\title{
Variability Aware Yield Optimal Sizing of Analog Circuits using SVM-Genetic Approach
}

\author{
D. Boolchandani, Lokesh Garg, Sapna Khandelwal and Vineet Sahula \\ Dept. of Electronics \& Comm. Engg. \\ National Institute of Technology \\ Jaipur, India. \\ Email: \{dbool,sahula\}@ieee.org, \{lokesh_garg20, sapna_k8\}@yahoo.co.in
}

\begin{abstract}
During analog circuit synthesis in nanometer technology, process variability analysis is mandatory during design space exploration. This would ensure that the circuit will function as per specifications after fabrication even with impact of statistical variations in nanometer regimes. The methodology necessitates the evaluation of performance metrics of an analog circuit for different sizing instances of the transistors. Circuit simulation for performance evaluation is very time consuming and is seldom a choice while sizing a circuit for a chosen topology. The complexity of sizing methodology increases with the need to consider effects of variations in process and environment parameters. We employ macromodeling approach for analog circuits based on support vector machine (SVM), which enables efficient evaluation of performance of such circuits during sizing and yield optimization loops. The objective to improve evaluation efficiency has been the motivation behind efforts to develop performance macromodels, which should be as accurate as SPICE and at the same time have shorter evaluation time for use in the sizing of analog circuits, where they are used as substitutes for full circuit simulation during circuit sizing (synthesis).
\end{abstract}

Process variability aware SVM macromodels are used in the multiobjective multivariate sizing method which is also yield optimal. Post design centering, the sized circuits will be able to provide functions as per specifications upon fabrication. Its application as process variability analysis tool is illustrated on two stage op amp and a voltage controlled oscillator using $90 \mathrm{~nm}$ BSIM4 models of transistors.

Keywords: Process variability analysis, Macromodels, Support Vector Machine, Yield, Design centering, Regression.

\section{INTRODUCTION}

The rapid scaling of CMOS technology has increased the significance and complexity of process variation. Process variation is the deviation of parameters from desired values due to limited controllability of a process. As MOS device size continues to rapidly scale down into the ultra deep submicron regime, reliability of manufacturing tools decreases while controlling design parameters. Random dopant fluctuation, annealing effects and lithographic limitation are some of the factors contributing to- wards process variation. These process variations manifest into variation of device parameters such as Threshold voltage, Oxide thickness, and Length of a transistor [1]. Variations in device parameters in turn affect performance metrics of analog circuits leading to loss in post design parametric yield. Yield is usually predicted by carrying out Monte Carlo analysis with circuit simulations for various process parameters. Process parameters are randomly and simultaneously varied according to their respective probability density function. Circuit performances are then evaluated for these multiple instances of process parameters. Because most circuits require very significant simulation time, the cost of performing a Monte Carlo analysis can be prohibitive except for small components. Analytical Modeling approaches [2] have been employed to model circuit performance as a function of process parameters. However, they compromise on accuracy. Machine learning approaches have been reported for macromodeling of analog circuits [3], [4]. These are performance macromodels, which can be trained using data generated directly from SPICE. They are build around suitable kernel functions as regression functions and are able to provide SPICE level accuracy. These SVM models can then be efficiently used inside Monte Carlo analysis loop to predict parametric yield. Process variability analysis tool so developed is used with Genetic Algorithm (GA) for Design centering of the performance parameters of the two stage op-amp and voltage controlled oscillator (VCO). GA is chosen for its empirical robustness in nonlinear and non convex objective functions. Authors in [5], present an approach for efficient yield optimization. It is based on specification-wise linearization of performance at the worst case points in the space of statistical parameters and linearization of the feasibility region. A robust coordinate search is reported, which performs better than gradient based algorithm resulting in improvement in nominal point and reduction in variance of circuit performance simultaneously. Linearization before search ensures that Monte-Carlo simulations are substantially re- 
duced for later computations.

The rest of the paper is organized as follows. We discuss design centering problem in Section II. Theory of SVM regression and yield analysis is presented in Section III. Proposed work and experimental setup are discussed in Section IV. Section V contains results. We conclude in Section VI.

\section{Design Centering Problem}

The objective of the design centering or yield maximization problem is to maximize the number of fabricated circuits whose performance meets a set of desired specifications. The parametric yield of a circuit as defined by authors in [6] is portion of the manufactured circuits that satisfies a set of acceptability constraints on performance defined by the user. The parametric yield $\mathrm{Y}$ of a circuit can be expressed as in (1). Here, $y$ is a vector of circuit performance features of interest (e.g., open loop gain, phase margin, etc), $f_{y}($.$) is the joint probability$ density function of $y$, and $a_{Y}$ is the output acceptability region in the $y$ space defined by acceptability constraints $y_{i}^{L} \leq y_{i} \leq y_{i}^{U}$, i.e., $a_{Y}=\left\{y_{i} \mid y_{i}^{L} \leq y_{i}^{U}\right\}$. We maximize the yield $Y$ over $D$, a feasible region of input parameters, $x \in D$. To save computational time inherent in SPICE we utilize macromodeling approach for analog circuits based on support vector machine, which provides efficient as well as accurate mapping between input design parameters and output performance features of such circuits.

$$
Y=\int_{a_{Y}} f_{y}(y) d y
$$

\section{SVM BASED YIELD ANALYSIS}

SVM regression have emerged as an efficient technique for modeling complex nonlinear relationships [4]. In the proposed design centering methodology, support vector models developed from SPICE simulation data are used for mapping input and output parameters of the circuit. We use extended SVM macromodel [7] which utilizes efficient kernels, instead of using conventional kernels. These models are then used to estimate yield, rather than performing a large number of time consuming evaluations by circuit simulators.

\section{A. SVM Macromodel}

Our work is based on the theory from [8]. Suppose we are given a training data $\left\{\left(x_{1}, y_{1}\right), \ldots\left(x_{k}, y_{k}\right)\right\} \subset$ $R^{N} \times R$, where $R^{N}$ represents input space. By a certain nonlinear mapping $\phi$, the training pattern $x_{t}$ is mapped into some feature space, in which a real valued function $y(x)$ is defined as in (2). Here, $\phi($.$) :$ $R^{n} \rightarrow R^{n} h_{h}$ is the mapping to the high dimensional and potentially infinite dimensional feature space. Given a training set $\left\{x_{k}, y_{k}\right\}_{k=1}^{N}$, optimization problem as in (3) is formulated in the primal weight space.

$$
y(x)=\omega^{T} \phi(x)+b \text { with } \omega \in R^{N}, b \in R
$$

$$
P: \min _{w, b, e} J_{p}(w, e)=\frac{1}{2} w^{T} w+\gamma \frac{1}{2} \sum_{k=1}^{N} e_{k}^{2}
$$

This formulation involves the trade off between a cost function term and a sum of squared errors governed by the trade-off parameter $\gamma$. In the regression formalism the term $\frac{1}{2} w^{T} w$ is no longer related to hyper-plane separation, but instead determines the smoothness of the resulting model. In fact, the primal problem in the LS-SVM formalism is wholly equivalent to a ridge regression problem formulated in the feature space, with parameter $\gamma$ performing the role of smoothing parameter. The dual Lagrangian-based formulation is given in (4), where $\mathcal{L}=J_{p}(w, e)-\sum_{k=1}^{N} \alpha_{k}\left\{w^{T} \phi\left(x_{k}\right)+b+e_{k}-y_{k}\right\}$ and $\alpha_{k}$ are Lagrange multipliers. Using the conditions for optimality $\frac{\partial \mathcal{L}}{\partial w}=0 \frac{\partial \mathcal{L}}{\partial b}=0 \frac{\partial \mathcal{L}}{\partial e_{k}}=0 \frac{\partial \mathcal{L}}{\partial \alpha_{k}}=0$ and eliminating variables $w$ and $e$, the resulting LSSVM model after applying kernel trick, for function estimation becomes as in (5). The function $K\left(x, x_{k}\right)$ corresponds to a dot product in the given feature space and satisfies the Mercer's conditions as discussed in Section III-B. SVM models are typically trained with a discrete set of data points called the training data set. This represent performance functionality of circuit being modeled.

$$
\begin{aligned}
& D: \max _{\alpha} \mathcal{L}(w, b, e ; \alpha) \\
& y(x)=\sum_{k=1}^{N} \alpha_{k} K\left(x, x_{k}\right)+b
\end{aligned}
$$

\section{B. Mercer kernel}

If the kernel $K$ is a symmetric positive definite function, which satisfies the Mercer's conditions as in equations (7) and (8), then the kernel $\mathrm{K}$ would represents an inner product in feature space as in equation (6)

$$
K\left(x_{k}, x\right)=\phi\left(x_{k}\right) \cdot \phi(x)
$$

and is known as Mercer Kernel.

$$
\begin{gathered}
K\left(x_{k}, x\right)=\sum_{i}^{\infty} a_{i} \phi_{i}\left(x_{k}\right) \phi_{i}(x), a_{i}>0 \\
\iint K\left(x_{k}, x\right) g\left(x_{k}\right) g(x) d x_{k} d x>0
\end{gathered}
$$

From these conditions the simple rules for composition of kernels can be concluded, which also satisfy Mercer's condition [9].

a) Combinations of kernels: : Let $k_{1}\left(x_{k}, x\right), k_{2}\left(x_{k}, x\right)$ be Mercer kernels and $c_{1}, c_{2} \geq 0$, then $k\left(x_{k}, x\right)=c_{1} k_{1}\left(x_{k}, x\right)+c_{2} k_{2}\left(x_{k}, x\right)$ and is also called a Mercer kernel. Moreover, the product of two Mercer kernels is a Mercer kernel, which is proved based on the equivalent definition of Mercer kernel. Similarly, it has been proposed in [10] that we can modify the kernel functions by multiplying it by a positive factor, adding bias, or taking exponential of the kernel. The new kernels so obtained are also 
Table I

LIST OF KERNELS WITH THEIR EXPRESSION

\begin{tabular}{c|l}
\hline Kernel composed & Kernel Expression \\
\hline \hline Linear kernel & $K\left(x, x_{j}\right)=x_{k}^{T} x$ \\
\hline RBF kernel & $K\left(x, x_{j}\right)=e^{\left(-\frac{\left\|x-x_{k}\right\|^{2}}{\sigma^{2}}\right)}$ \\
\hline Hybrid kernel & $K\left(x, x_{j}\right)=e^{-\frac{\left\|x-x_{k}\right\|^{2}}{\sigma^{2}}} \times\left(\tau+x_{k}^{T} x\right)^{d}$ \\
\hline Multiplied kernel & $K\left(x, x_{k}\right)=a \times k\left(x, x_{k}\right)$ where $a>0$ \\
\hline Power kernel & $K\left(x, x_{k}\right)=-\left\|x-x_{k}\right\|^{\beta} 0<\beta \leq 1$ \\
\hline Log kernel & $K\left(x, x_{k}\right)=-\log \left(1+\left\|x-x_{k}\right\|^{\beta}\right) 0<\beta \leq 1$ \\
\hline
\end{tabular}

a Mercer Kernel. The kernel that is applied in the present work is $\log$ Kernel [11], which alongwith other kernels are given in Table I. All these kernels satisfy the Mercer's condition, which is necessary for the problem to be convex, and hence providing unique and optimum solution.

\section{Design Centering}

The proposed design centering methodology has two steps. Step 1 is the parametric yield estimation stage wherein yield is estimated by performing Monte Carlo simulations using SVM regression models. In the second step, the parametric yield estimator is coupled with GAs to facilitate the search for the design center that provides the greatest yield. The circuit under consideration is two stage op amp and $\mathrm{VCO}$.

1) Parametric Yield Estimation: The parametric yield estimation step begins with a random sample generator that uses Monte Carlo runs to generate a large number of input vectors based on the mean, variance, and distribution of the input variables. Examples of input variables could be process as well as design parameters viz. gate length, oxide thickness, threshold voltage of MOSFETs of the circuits. Output performance features that could be considered are open-loop gain, phase margin and unity gain frequency. Parametric yield is calculated based on the specification for each output performance feature.

2) Genetic Design Centering: In this stage, the parametric yield estimator is coupled with a genetic scheme for design centering. The values obtained from the parametric yield estimator are used in conjunction with Genetic algorithm (GA) to determine the mean of the input parameters that result in the maximum parametric yield.

\section{EXPERIMENTAL SETUP}

The two-stage op amp and voltage-controlledoscillator (VCO) as shown in Figure 1, have been chosen to illustrate design centering for sizing. Authors have shown in [1] that relatively few device variables have capability to capture the bulk of the variations in a MOS manufacturing line with the most significant ones being threshold voltage, oxide thickness variation and the length and width lithographic variations. For analog cells, the device widths are much larger than lengths, so circuit performances are more sensitive to length variations than to width variations. As result three sources of variation, oxide thickness $\triangle t_{o x}$, threshold voltage variation $\triangle V_{t h}$ and channel length variation $\triangle L$ dominate most analog designs. These device variables exhibit two types of tolerances, global and local. Global parameter tolerances are due to chipto-chip (inter-chip) and wafer-to-wafer fluctuation of the manufacturing process. They are modeled as parameter variations that affect all transistors of a circuit in the same way, hence the notation global. Whereas the local parameter tolerances are due to variations within a chip (intra-chip) and that affects transistors individually and independently. They lead to different behaviors of transistors in a circuit, a so-called mismatch. Performance features of analog circuits that have transistor pairs are very sensitive to such mismatch.

In modern processes, oxide thickness variation is tightly controlled and it has global variation and so all the transistors of two circuits are assumed to have same variation in oxide thickness [12]. However, transistor threshold voltages and channel lengths have both global as well as local variations. We use $90 \mathrm{~nm}$ BSIM4 Model [13] for the transistors in circuit. For this technology, $3 \sigma$ global variations in oxide thickness, threshold voltage and channel length of transistor are adapted from [14] and are reproduced for op amp as well as for VCO in Table II. Similarly, the design parameters and performance specification for VCO are given in Table IV. Whereas $3 \sigma$ local variations in threshold voltage and channel length of transistors are taken from [15] and is given in Table II. They are presumed to have Gaussian distribution [16]. The global and local variations in device parameters lead to variations in output performance features of two stage op amp and VCO. HSPICE is used to evaluate various performance features related to circuit under consideration influenced by variability of device parameters. A set of 4000 data correlating device parameters variability with performance features is generated using HSPICE. SVM macromodel is then trained on them using Least square SVM toolbox [17] interfaced with MATLAB. Once we have obtained the SVM macromodels for different output performance features of two stage op amp circuit, we use them to estimate parametric yield of the circuit. The parametric yield estimate for the given circuit is given as $Y=Y_{G} \cap Y_{U G F} \cap Y_{P H M}$. Here, $Y_{G}=$ yield due to open loop gain, $Y_{U G F}=$ yield due to unity gain frequency and $Y_{P H M}=$ yield due to Phase margin. The combined yield is obtained by calculating the number of times all output performance features of circuit satisfy the specifications limit given in Tables III and Table IV for varia- 


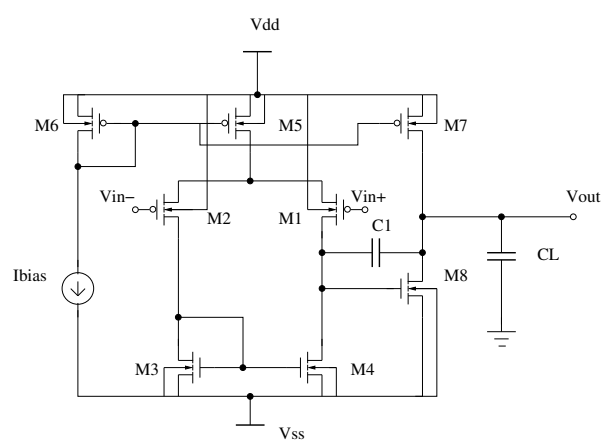

(a)

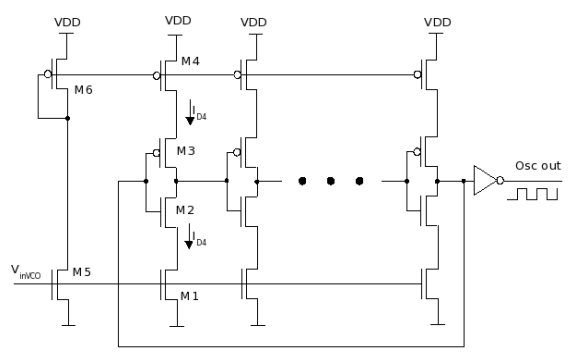

(b)

Figure 1. (a) Two Stage op-amp circuit and (b) Voltage controlled oscillator circuit (ring oscillator based)

Table II

DESIGN PARAMEter VAlues For BOTh the CIRCUITS

\begin{tabular}{c|c|c|c}
\hline $\begin{array}{c}\text { Design \& } \\
\text { process } \\
\text { parameters }\end{array}$ & $\begin{array}{c}\text { Mean } \\
\text { value }\end{array}$ & $\begin{array}{c}3 \sigma \\
\text { Global } \\
\text { variation }\end{array}$ & $3 \sigma$ Local variation \\
\hline \hline$L$ & $0.5 \mu m$ & $12 \%$ & $\frac{3 \times A_{L}}{(W)^{1 / 2}} \mu m$ \\
\hline$t_{o x}$ & $1.4 n m$ & $2 \%$ & no local variations \\
\hline$V_{t h n}$ & $0.397 V$ & $16 \%$ & $\frac{3 \times A_{V T N}}{(2 \times W \times L)^{1 / 2}} m V$ \\
\hline$V_{t h p}$ & $-0.339 V$ & $16 \%$ & $\frac{3 \times A_{V T P}}{(2 \times W \times L)^{1 / 2}} m V$ \\
\hline
\end{tabular}

tion in device parameters. Optimization of yield for above cases is done using Genetic approach, through adjustment of mean values of device and process parameters given in Table II. The Genetic Algorithm parameters chosen are- crossover probability (0.8), mutation probability (0.01), population size (20) and number of generations (100).

Table III

SPECIFICATION OF OUTPUT PERFORMANCE FEATURES FOR OP AMP

\begin{tabular}{c|c|c}
\hline $\begin{array}{c}\text { Open loop Gain } \\
(\boldsymbol{G})\end{array}$ & $\begin{array}{c}\text { Unity gain } \\
\text { Frequency }(\boldsymbol{U G F})\end{array}$ & $\begin{array}{c}\text { Phase Margin } \\
(\boldsymbol{P H M})\end{array}$ \\
\hline$\geq 40 \mathrm{~dB}$ & $\geq 4 M h z$ & $\geq 45^{\circ}$ \\
\hline
\end{tabular}

Table IV

DESIGN PARAMETER AND SPECIFICATIONS FOR VCO

\begin{tabular}{|c|c|}
\hline MOSFET & W/L values \\
\hline \hline PMOS & $4.5 / 0.19(\mu \mathrm{m})$ \\
\hline NMOS & $1.5 / 0.19(\mu \mathrm{m})$ \\
\hline
\end{tabular}$\quad$\begin{tabular}{c|c|} 
Center frequency \\
\hline $340 \mathrm{MHz} \geq f_{0} \geq 180 \mathrm{MHz}$ \\
\hline
\end{tabular}

\section{REsults}

Correlation coefficients have been evaluated between the output data generated by HSPICE and the SVM models for different cases and shown in Table $\mathrm{V}$, and some of them are illustrated in Fig. . In all the cases, the correlation coefficient is found to be close to unity indicating high HSPICE like accuracy of the SVM model. For illustration of speed-up when we use SVM macromodel, the time taken for performance evaluation by SVM macromodel as well as by HSPICE is shown in Table VI. We find SVM models to be faster by an order of two. In fact, the time spent in training the SVM macromodel with 4000 data tuple is $T_{\text {training }}=20$ sec. SVM macromodel once trained, is then ready for being used repeatedly for performance evaluation. We use SVM macromodel inside Monte-Carlo loop while computing yield, which required 4000 data tuples for effecting variability. Time taken by this yield analysis methodology is $T_{\text {yield }}=21$ sec. The total time taken by genetic algorithm for variability aware sizing using similar SVM based yield-analysis methodology, is $T_{\text {yield-aware-sizing }}=838$ sec. using 100 generations. Had the HSPICE been used in place of SVM model, we contemplate that $T_{\text {yield(HSPICE) }}$ would be $20 \times$ that of $T_{\text {yield }}$, and subsequently $T_{\text {yield-aware-sizing }(H S P I C E)}$ would be approximately $20 \times$ of $T_{\text {yield-aware-sizing }}$.

Parametric yield estimation using SVM macromodel before optimization is shown in Figure 2 and 5(a). The yield is then optimized using SVM-GA based design centering and is shown in Figures 3 and 5(b). From Table VII we observe that yield has improved considerably.

Table V

CORRELATION COEFFICIENTS BETWEEN OUTPUT PERFORMANCE GENERATED BY HSPICE AND SVM MODELS

\begin{tabular}{c|c|c|c}
\hline \multirow{2}{*}{$\begin{array}{c}\text { Design } \\
\text { Parameter }\end{array}$} & \multicolumn{3}{|c}{ Performance Parameter } \\
\cline { 2 - 4 } & Gain & $\boldsymbol{P H M}$ & $\boldsymbol{U} \boldsymbol{G F}$ \\
\hline \hline$L$ & 0.9780 & 0.9839 & 0.9867 \\
\hline$V_{t h}$ & 0.9797 & 0.9865 & 0.9829 \\
\hline$t_{\text {ox }}$ & 0.9951 & 0.9370 & 0.9988 \\
\hline
\end{tabular}



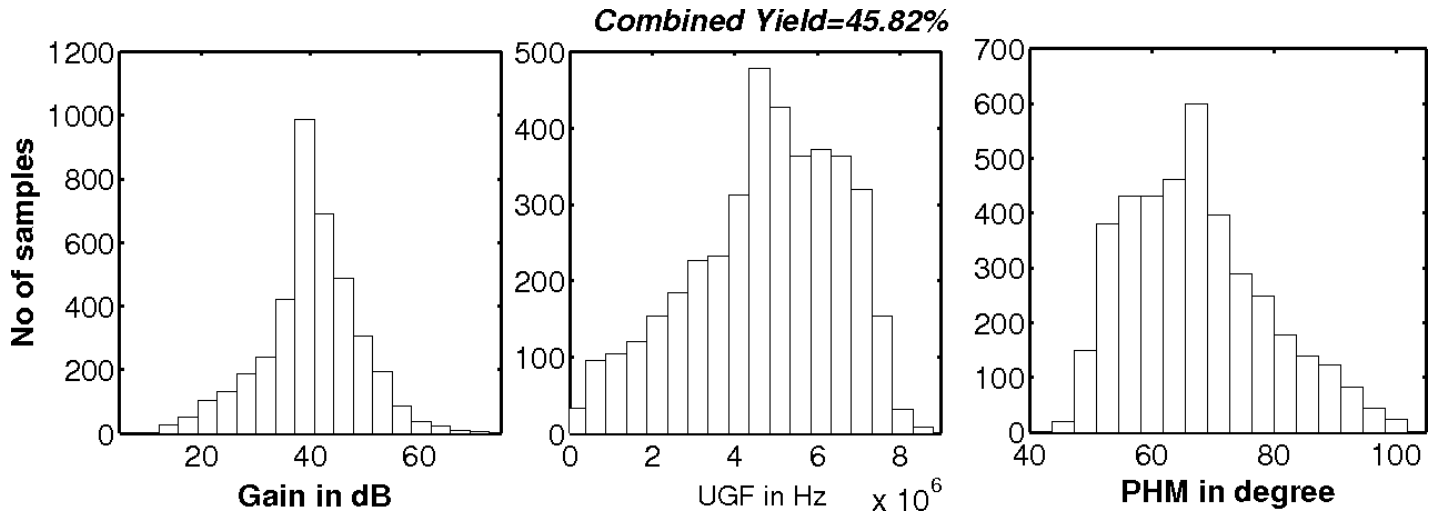

Figure 2. Histograms for performance parameters and combined yield due to variations in $L$, and $V_{t h}$ without design centering
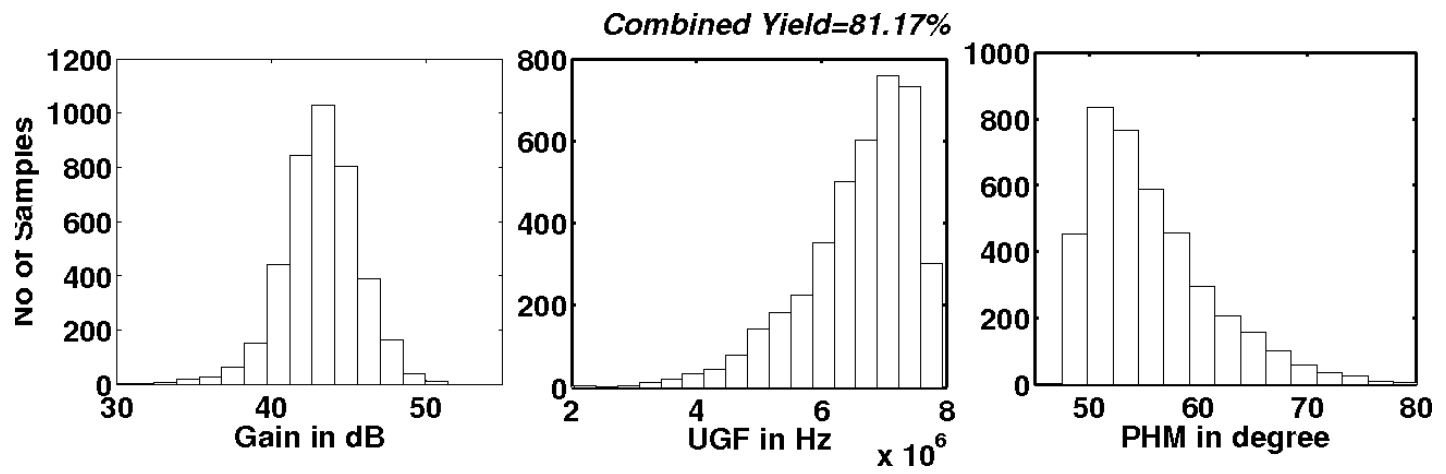

Figure 3. Histograms for performance parameters and combined yield due to variations in $L$, and $V_{t h}$ with design centering

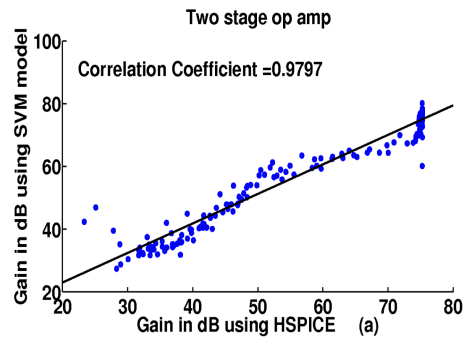

(a)

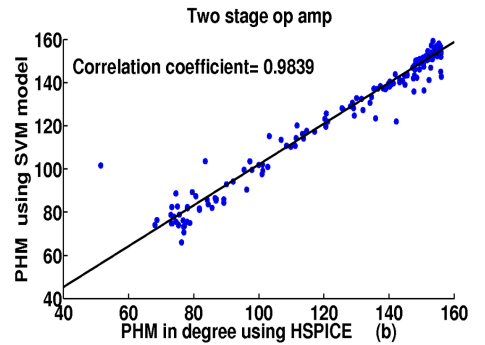

(b)

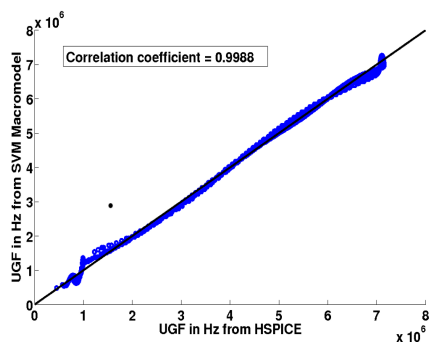

(c)

Figure 4. Scatter plots illustrating closeness of correlation-coefficients to Unity

Table VI

PERFORMANCE EVALUATION TIME OF HSPICE AND SVM MACROMODEL FOR 500 DATA SETS

\begin{tabular}{c|c|c|c|c}
\hline $\begin{array}{c}\text { Performance } \\
\text { feature }\end{array}$ & Variations & $\begin{array}{c}T_{H S P I C E} \\
(\mathbf{s e c})\end{array}$ & $\begin{array}{c}T_{\text {macromodel }} \\
\text { (sec) }\end{array}$ & $\begin{array}{c}\text { Speed } \\
\text { up }\end{array}$ \\
\hline \hline Gain & $\triangle L$ & 14.2 & 0.63 & 22 \\
\hline UGF & $\triangle L$ & 14.2 & 0.63 & 22 \\
\hline PHM & $\triangle L$ & 14.2 & 0.63 & 22 \\
\hline Gain & $\triangle V_{t h}$ & 14.1 & 0.64 & 22 \\
\hline UGF & $\triangle V_{t h}$ & 14.1 & 0.64 & 22 \\
\hline PHM & $\triangle V_{t h}$ & 14.1 & 0.63 & 22 \\
\hline$f_{0}$ & $\triangle V_{t h}$ & 79.0 & 3.0 & 26 \\
\hline
\end{tabular}

\section{CONCLUSIONS}

A process-aware SVM Model has been developed, which can link the process parameter variations to
Table VII

YIELD IMPROVEMENT AFTER MULTIOBJECTIVE Multivariate DESIGN CENTERING FOR VARIABILITY IN ALL DESIGN PARAMETERS

\begin{tabular}{c|c|c|c}
\hline Circuit & $\begin{array}{c}\text { Design \& } \\
\text { process } \\
\text { parame- } \\
\text { ters }\end{array}$ & $\begin{array}{c}\text { Unoptimized } \\
\text { combined } \\
\text { yield (\%) }\end{array}$ & $\begin{array}{c}\text { Optimized } \\
\text { combined } \\
\text { yield (\%) }\end{array}$ \\
\hline \hline Op amp & $L, t_{\text {ox }} \& V_{t h}$ & $45.8 \%$ & $81.2 \%$ \\
\hline $\mathrm{VCO}$ & $L \& V_{t h}$ & $50.5 \%$ & $99.2 \%$ \\
\hline
\end{tabular}

performance parameters for analog circuits. Applicability of the model during sizing has been illustrated by performing yield analysis with process variability using an example circuits i.e., two stage op-amp and VCO. The model has been found to be highly efficient and is close in accuracy to SPICE. It has 


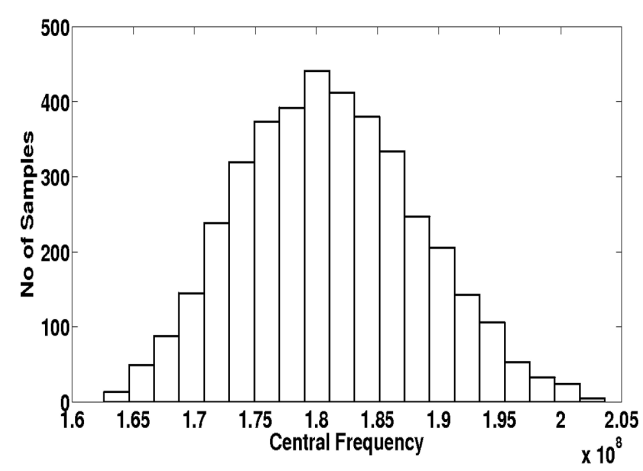

(a)

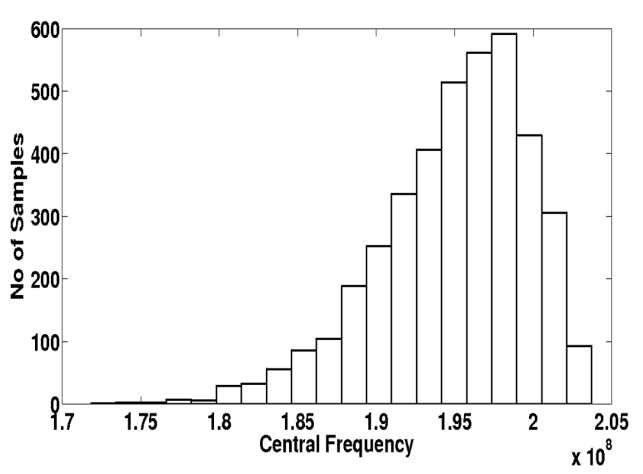

(b)

Figure 5. Histograms for center-frequency of VCO- (a) Un-optimized and (b) optimized, combined yield

been found that Monte Carlo analysis using SVM model based evaluation is much faster than SPICE based evaluation with almost similar accuracy. The effects of variation in channel length and threshold voltage on the yield were observed to be significant. The process of design centered sizing has been illustrated using Genetic Algorithm. Yield has been improved considerably and thus the impact of process variations has been minimized during sizing for two-stage op-amp and VCO circuits using $90 \mathrm{~nm}$ technology.

\section{REFERENCES}

[1] L. T. Mukherjee and R. Rutenbar, "Efficient handling of operating range and manufacturing line variations in analog cell synthesis," in IEEE Trans. Computer-Aided Design, vol. $19,2000$.

[2] C. Kurker, J. Paulos, R. Gyurcsik, and J.-C. Lu, "Hierarchical yield estimation of large analog integrated circuits," in IEEE Journal of Solid-State Circuits, vol. 28, pp. 203-209, Mar 1993.

[3] T. Kiely and G. G. E. Gielen, "Performance modeling of analog integrated circuits using least-squares support vector machines," in Proceedings of DATE, pp. 448-453, 2004.

[4] M. Ding and R. Vemuri, "A combined feasibility and performance macromodel for analog circuits," in Proceedings of IEEE Design Automation Conference, pp. 63-68, 2005.

[5] F. Schenkel, M. Pronath, S. Zizala, R. Schwencker, H. Graeb, and K. Antreich, "Mismatch analysis and direct yield optimization by spec-wise linearization and feasibilityguided search," in Design Automation Conference, 2001. Proceedings, pp. 858 - 863, 2001.

[6] R. Pratap, P. Sen, C. Davis, R. Mukhophdhyay, G. May, and J. Laskar, "Neurogenetic design centering," in IEEE Transactions on Semiconductor Manufacturing, vol. 19, pp. 173182, May 2006.

[7] D. Boolchandani, A. Ahmed, and V. Sahula, "Efficient kernel functions for support vector machine regression model for analog circuits' performance evaluation," Analog Integrated Circuits and Signal Processing, pp. 1-12, 2010. 10.1007/s10470-010-9476-6.
[8] V. Vapnik, The Nature of Statistical Learning Theory. Springer-Verlag, NY USA, 1995.

[9] G. Smits and E. Jordaan, "Improved svm regression using mixtures of kernels," in Proceedings of International Joint Conference on Neural Networks, vol. 3, pp. 2785-2790, 2002.

[10] J. A. Suykens, T. Gestel, J. Brabenter, B. Moor, and J. Vandewalle, Least Square Support vector Machines. World Scientific Publishing Co. Pte. Ltd, 2002.

[11] S. Boughorbel, J.-P. Tarel, and N. Boujemaa, "Conditionally positive definite kernels for svm based image recognition," in Proceedings of ICME, pp. 113-116, 2005.

[12] H. E. Graeb, "Analog design centering and sizing," Springer Publishing Co., 2007.

[13] BSIM Homepage - Offical Site, http://wwwdevice.eecs.berkeley.edu/ bsim3/bsim4.

[14] ITRS, "International Techology Roadmap for Semiconductors," tech. rep., 2007.

[15] W. M. Sansen, Analog Design Essentials. Springer, 2006.

[16] M. Pelgrom, A. Duinmaijer, and A. Welbers, "Matching properties of MOS transistors," IEEE Journal of Solid-State Circuits, vol. 24, pp. 1433-1439, Oct 1989.

[17] J. A. Suykens, "Least squares support vector machine matlab/c toolbox. http://www.esat.kuleuven.be/sista/lssvmlab," 2003.

[18] M. Barros, J. Guiherme, and N. Horta, "Ga-SVM feasibility model and optimization kernel applied to analog ICs design automation," in Proc. $17^{\text {th }}$ ACM Great Lake Symposium on VLSI, USA, pp. 469-472, 2007.

[19] X. Li and L. Pileggi, "Efficient parametric yield extraction for multiple correlated non-normal performance distributions of analog/RF circuits," in Proc. IEEE ACM Design automation Conference, USA, 2007.

[20] T. McConaghy and G. Gielen, "Globally reliable variationaware sizing of analog integrated circuits via response surfaces and structural homotopy," IEEE Trans. on CAD of ICs and Systems, vol. 28, pp. 1627-1640, Nov. 2009.

[21] S.K.Tiwary, P.K.Tiwary, and R. A. Rutenbar, "Generation of yield-aware pareto surfaces for hierarchical circuit design space exploration," in Proceedings of IEEE Design Automation Conference, pp. 31-36, 2006.

[22] M. Hershenson, S. Boyd, and T. Lee, "Optimal design of a CMOS op-amp via geometric programming," IEEE Transactions on Computer-Aided Design of Integrated Circuits and Systems, vol. 20, pp. 1-21, Jan 2001. 